\title{
Plasma Rivaroxaban Level to Identify Patients at Risk of Drug Overexposure: Is a Single Measurement of Drug Level Reliable?
}

\author{
Krishnan Shyamkumar ${ }^{1}$ Jack Hirsh ${ }^{2}$ Vinai C. Bhagirath ${ }^{1,2,3}$ Jeffrey S. Ginsberg ${ }^{2}$ \\ John W. Eikelboom ${ }^{1,2,3}$ Noel C. Chan ${ }^{1,2,3}$
}

1 Population Health Research Institute, Hamilton, Ontario, Canada
2 Division of Hematology and Thromboembolism, Department of
Medicine, McMaster University, Hamilton, Ontario, Canada
${ }^{3}$ Thrombosis and Atherosclerosis Research Institute, Hamilton,
Ontario, Canada

TH Open 2021;5:e84-e88.
Address for correspondence Noel C. Chan, MBBS, DBCVRI, 237

Barton St East, Hamilton, ON L8L 2X2, Canada

(e-mail: noel.chan@taari.ca).

\begin{abstract}
Keywords

- rivaroxaban

- drug levels

- NOACs

- atrial fibrillation

- venous

thromboembolism

Introduction Dose adjustment based on laboratory monitoring is not routinely recommended for patients treated with rivaroxaban but because an association has been reported between high drug level and bleeding, it would be of interest to know if measuring drug level once could identify patients at risk of bleeding who might benefit from a dose reduction. Objective This study was aimed to investigate the reliability of a single measurement of rivaroxaban level to identify clinic patients with persistently high levels, defined as levels that remained in the upper quintile of drug-level distribution.

Methods In this prospective cohort study of 100 patients with atrial fibrillation or venous thromboembolism, peak and trough rivaroxaban levels were measured using the STA-Liquid Anti-Xa assay at baseline and after 2 months. Values of 395.8 and $60.2 \mathrm{ng} / \mathrm{mL}$ corresponded to the 80th percentile for peak and trough levels, respectively, and levels above these cut-offs were categorized as high for our analyses.

Results Among patients with a peak or trough level in the upper quintile at baseline, only $26.7 \%$ (95\% confidence interval [CI]: $10.9-52.0 \%)$, and $13.3 \%(95 \% \mathrm{Cl}: 2.4-37.9 \%$ ), respectively, remained above these thresholds.

Conclusion Our findings do not support the use of a single rivaroxaban level measurement to identify patients who would benefit from a dose reduction because such an approach is unable to reliably identify patients with high levels.
\end{abstract}

\section{Introduction}

Based on their convenience and favorable results in clinical trials, the direct oral anticoagulants (DOACs) are replacing vitamin-K antagonists for various clinical indications. ${ }^{1,2}$ Rivaroxaban was the first oral factor Xa inhibitor licensed for stroke prevention in atrial fibrillation (AF) and for venous thromboembolism (VTE) prevention and treatment. ${ }^{3}$ Like the other DOACs, drug levels of rivaroxaban vary among patients and

received

September 2, 2020

accepted after revision

November 5, 2020
DOI https://doi.org/

$10.1055 / \mathrm{s}-0040-1721734$.

ISSN 2512-9465. there is indirect evidence from a subanalysis of the ROCKET-AF trial that the risk of bleeding correlates with rivaroxaban levels as measured by the prothrombin time. ${ }^{4}$ Based partly on these data, it has been suggested that measuring drug levels would be useful for identifying patients who might benefit from a dose reduction. ${ }^{5-7}$ However, dose adjustment based on drug level measurement would only be practical if the dose response to rivaroxaban did not vary over time. In this report, we examine the possibility that an approach based on a single

\section{(c) 2021. The Author(s).}

This is an open access article published by Thieme under the terms of the Creative Commons Attribution License, permitting unrestricted use, distribution, and reproduction so long as the original work is properly cited. (https://creativecommons.org/licenses/by/4.0/)

Georg Thieme Verlag KG, Rüdigerstraße 14, 70469 Stuttgart, Germany 
random measurement of peak or trough rivaroxaban level can be used to identify patients with persistently high levels who might benefit from a dose reduction.

\section{Methods}

\section{Study Design}

Present study is a prospective observational study of 100 patients. The study protocol was reviewed and approved by the Hamilton Integrated Research Ethics Board.

\section{Patients}

Consecutive adult patients with AF (permanent, paroxysmal, or persistent) or VTE (idiopathic or provoked) receiving longterm rivaroxaban therapy were enrolled from outpatient clinics at the Hamilton General Hospital. Eligible patients who were either geographically inaccessible for follow-up, or unwilling or unable to provide written informed consent, were excluded.

\section{Follow-up}

Patients were seen at baseline and 2 months after enrolment with trough and peak rivaroxaban levels measured at each visit. As this was a noninterventional study to evaluate interand intraindividual variability of rivaroxaban levels with the current dosing scheme, clinicians were blinded to the results of these measurements.

\section{Collection and Processing of Blood Samples}

Attempts were made to measure rivaroxaban level at steady state. This meant that patients had to be receiving once daily rivaroxaban for at least 1 week before blood sampling, and for patients with VTE, we included only levels measured when treated with rivaroxaban 20-mg daily. Blood samples for obtaining trough drug levels were collected 24 hours after the last dose of rivaroxaban. Patients were then directed to take their next daily dose, and blood samples for obtaining peak drug levels were collected 2.5 hours later. At each time point, $10 \mathrm{~mL}$ of blood was collected into Becton Dickinson Vacutainer tubes (Becton Dickinson, Mississauga, Ontario, Canada), containing 3.2\% buffered trisodium citrate (9:1, $\mathrm{vol} / \mathrm{vol}$ ) by a trained research assistant. Immediately after collection, the tube was inverted three to five times, cellular elements were sedimented by twice subjecting the sample to centrifugation at $1,700 \times g$ for 15 minutes at $23^{\circ} \mathrm{C}$, and the resultant platelet-poor plasma was then harvested and stored in $1-\mathrm{mL}$ aliquots at $-80^{\circ} \mathrm{C}$.

\section{Antifactor Xa Activity Assay}

Plasma anti-Xa activity was measured using the STA-Liquid Anti-Xa assay as per the manufacturer's instructions, on the STA-R platform (Diagnostica Stago, Ansières-sur-Seine, France). Rivaroxaban concentration was estimated by referring to a calibration curve constructed with rivaroxaban calibrators (Diagnostica Stago, Ansières-sur-Seine, France). The limit of detection was $30 \mathrm{ng} / \mathrm{mL}$, and this value was imputed for the purpose of statistical analysis, whenever patients had levels below the limit. The inter- and intra-assay coefficients of variation (CVs) are reported to be 1.0 to 4.3 and 0.8 to $3.3 \%$, respectively, lower than the expected intraand interpatient CVs of rivaroxaban level. ${ }^{8}$

\section{Statistical Analyses}

Descriptive statistics were used to summarize the data. Normally distributed data were described using means and standard deviation if normally distributed and median and interquartile range if not. Categorical data were reported as proportions with 95\% confidence intervals (CIs), calculated using the Wald method. To assess the reliability of a single measurement to identify patients with a high rivaroxaban level, we use the 80th percentile of baseline peak and trough drug level distribution as cut-offs and calculate the proportions (and $95 \% \mathrm{CI}$ ) of patients whose levels remained high at the subsequent visit. Values of 395.8 and $60.2 \mathrm{ng} / \mathrm{mL}$ corresponded to the 80th percentile for peak and trough levels, respectively, and levels above these cut-offs were categorized as high for our analyses. Intrapatient variability of rivaroxaban in patients with an initial high drug levels was further explored by examining the proportions (and 95\% CIs) of drug levels that fell below the median level on subsequent measurements. These analyses were performed independently for peak and trough levels.

\section{Results}

-Fig. 1 summarizes the follow-up of recruited patients and the number of plasma rivaroxaban levels available for analysis at each visit. Of the 100 patients enrolled, 10 patients were unwilling or unable to attend the second visit, 9 had rivaroxaban discontinued, and 2 could not be contacted for the follow-up visit. A total of 79 patients with levels measured at both visits were included in the intrapatient variability analysis. Results for peak plasma levels were available at baseline and month 2 for 98 and 79 patients, respectively, and those for trough levels were available for 100 and 78 patients, respectively. Blood for trough levels was collected at a median (interquartile range [IQR]) of 24.8 (23.8-25.8) hours while blood for peak levels was collected at 2.5 (2.4-2.5) hours after rivaroxaban ingestion.

\section{Baseline Characteristics}

The baseline characteristics of the entire cohort and the analysis population (i.e., the subgroup of patients for whom drug levels were measured at both baseline and month 2) are presented in - Table 1. The mean age and weight of the entire cohort were 64.7 years and $90.1 \mathrm{~kg}$, respectively, and the median creatinine clearance was $97.9 \mathrm{~mL} / \mathrm{min}$. About $64 \%$ of these patients were male, and the proportion of patients taking rivaroxaban for AF was $51 \%$. The baseline characteristics of the analysis population were similar to those of the entire cohort.

\section{Ranges for Peak and Trough Levels}

For the entire population, median peak levels (p10 and p90) at baseline and month 2 were 280.5 (159.6-419.56) and 252 (127-458) ng/mL, respectively, whereas median trough levels (p10 and p90) at baseline and month 2 were 43.2 (28.1-90) and 35.5 (25-62.1) ng/mL, respectively. Among patients with 


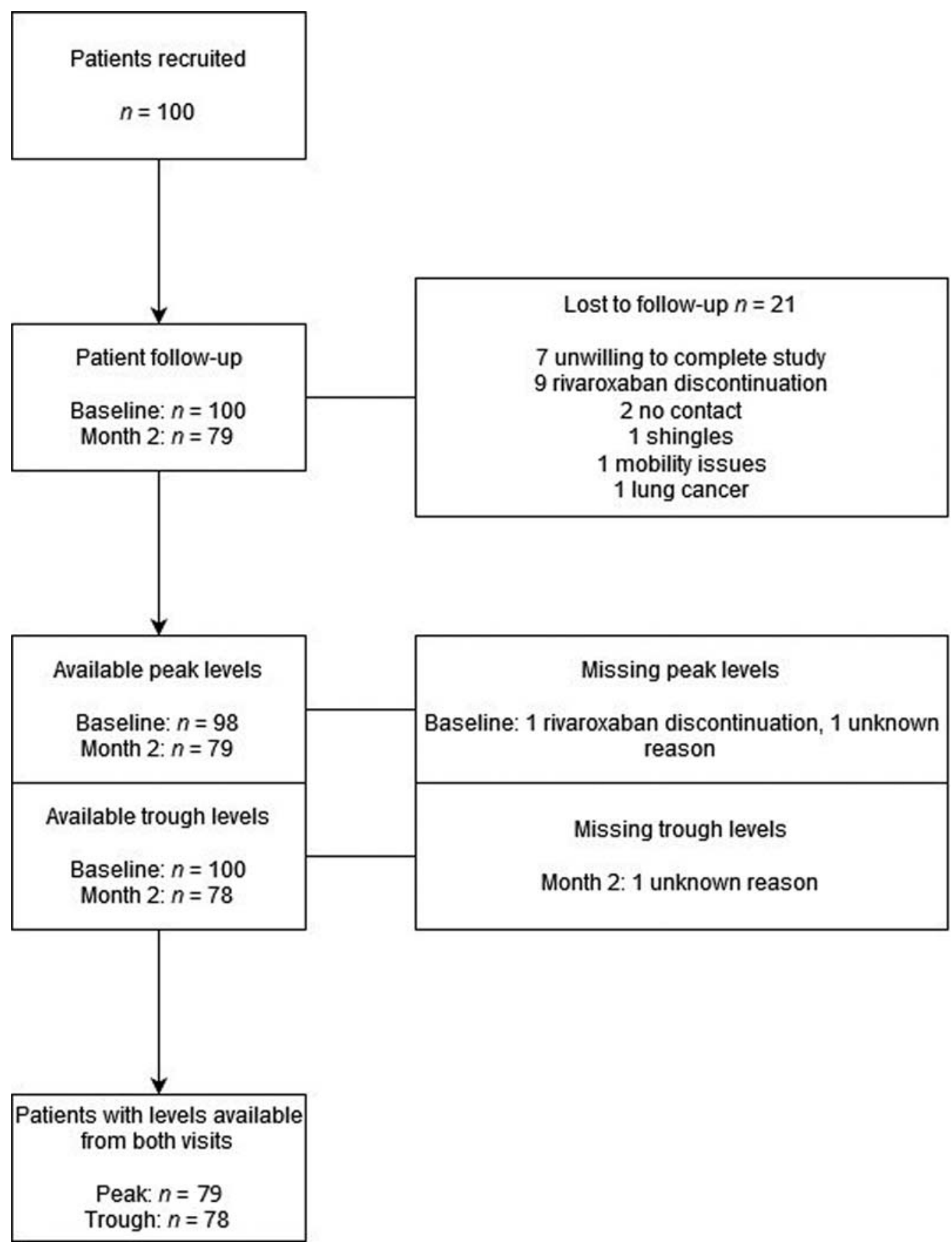

Fig. 1 Patient recruitment, follow-up and availability of test results.

rivaroxaban measurements performed at both visits, the median peak levels (p10 and p90) at baseline and month 2 were 278 (159.6-419.3) and 251 (126.8-460) ng/mL, respectively, and the corresponding trough levels (p10 and p90) were 43 (29.5-91.3) and $35.5(25-62.1) \mathrm{ng} / \mathrm{mL}$, respectively.

\section{Intrapatient Variability of Rivaroxaban in Patients with High Drug Levels}

Peak levels

- Fig. 2 shows the change in peak level over time in patients whose initial levels fell in the upper quintile of the drug level distribution ( $\geq 395.8 \mathrm{ng} / \mathrm{mL}$ ). Of these patients, only $26.7 \%$ (95\% CI: 10.9-52.0\%) had levels that remained above this threshold, and approximately $40.0 \%$ (95\% CI: 19.8-64.3\%) had levels that fell below the median peak level at month 2 ( - Table 2).

\section{Trough Levels}

- Fig. 3 shows the change in trough level over time in patients whose initial levels fell in the upper quintile of the distribution ( $\geq 60.2 \mathrm{ng} / \mathrm{mL}$ ). Of these patients, $13.3 \%$ (95\% CI: 2.4-37.9\%) had levels that remained above this threshold, while $33.3 \%$ (95\% CI: 15.2-58.3\%) had levels that fell below the median baseline level at month 2 (-Table 2 ). 
Table 1 Baseline characteristics of the entire study population and the analysis population

\begin{tabular}{|l|l|l|}
\hline & $\begin{array}{l}\text { All patients } \\
(\boldsymbol{n}=100)\end{array}$ & $\begin{array}{l}\text { Analysis } \\
\text { population } \\
(\boldsymbol{n}=79)^{\mathrm{a}}\end{array}$ \\
\hline $\begin{array}{l}\text { Age (y) } \\
\text { Mean } \pm \text { SD }\end{array}$ & $64.7 \pm 13.0$ & $65.4 \pm 12.3$ \\
\hline $\begin{array}{l}\text { Male sex } \\
n(\%)\end{array}$ & $64(64.0)$ & $50(63.3)$ \\
\hline $\begin{array}{l}\text { Weight (kg) } \\
\text { Mean } \pm \text { SD }\end{array}$ & $90.1 \pm 20.5$ & $90.2 \pm 20.9$ \\
\hline $\begin{array}{l}\text { Weight 50-100 kg } \\
n(\%)\end{array}$ & $75(75.0)$ & $59(74.7)$ \\
\hline $\begin{array}{l}\text { Weight }>100 \mathrm{~kg} \\
n(\%)\end{array}$ & $25(25.0)$ & $20(25.3)$ \\
\hline $\begin{array}{l}\text { CrCl (mL/min) } \\
\text { Median (range) }\end{array}$ & 97.9 & 94.5 \\
\hline $\begin{array}{l}\text { Indications } \\
n(\%)\end{array}$ & $(32.0-283.5)$ & $42.0-206.4)$ \\
\hline Atrial fibrillation & $51(51.0)$ & $40(51.3)$ \\
\hline $\begin{array}{l}\text { Venous } \\
\text { thromboembolism }\end{array}$ & $49(49.0)$ & $39(48.7)$ \\
\hline $\begin{array}{l}\text { Dosing } \\
n \text { (\%) }\end{array}$ & $91(91.0)$ & $49.1)$ \\
\hline $\begin{array}{l}20 \text { mg once daily } \\
15 \text { mg once daily }\end{array}$ & $9(9.0)$ & \\
\hline
\end{tabular}

Abbreviations: $\mathrm{CrCl}$ : creatinine clearance estimated using the Cockcroft-Gault formula; SD, standard deviation.

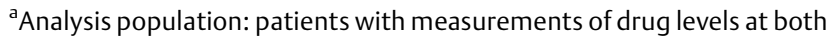
visits.

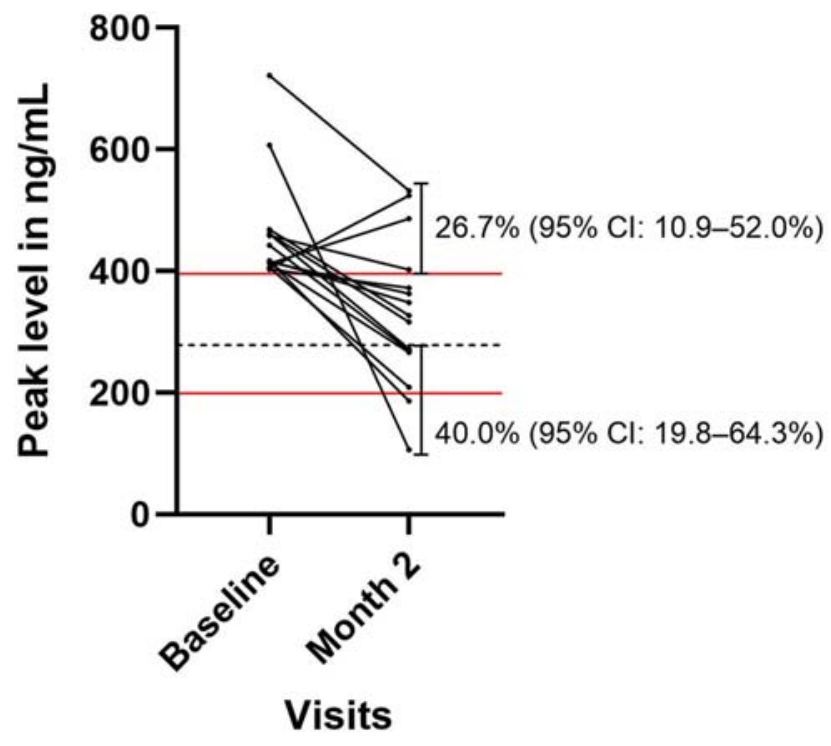

Fig. 2 Change in peak rivaroxaban levels across visits in patients with levels falling in the upper 20 th percentiles at baseline $(\geq 395.8 \mathrm{ng} / \mathrm{mL})$. Solid, red horizontal lines represent the 20th and 80th percentiles of peak levels observed at baseline. Dashed, black horizontal lines represent the median peak level at baseline $(278.0 \mathrm{ng} / \mathrm{mL})$. Proportions of patients with levels remaining above or below the extreme 20th percentiles at month 2 are shown by the respective vertical capped lines.
Table 2 Intraindividual variability of rivaroxaban levels in patients with an initial high level

\begin{tabular}{|l|l|l|}
\hline & Peak levels $^{\mathbf{a}}$ & Trough levels $^{\mathbf{b}}$ \\
\hline No of patients (\%) with & $4 / 15$ & $2 / 15$ \\
persistently high levels & $26.7 \%$ & $13.3 \%$ \\
on repeat measurement & $(95 \% \mathrm{Cl}:$ & $(95 \% \mathrm{Cl}:$ \\
& $10.9-52.0 \%)$ & $2.4-37.9 \%)$ \\
\hline No of patients (\%) with & $6 / 15$ & $5 / 15$ \\
levels below the median & $40.0 \%$ & $33.3 \%$ \\
on repeat measurement & $(95 \% \mathrm{Cl}:$ & $(95 \% \mathrm{Cl}:$ \\
& $19.8-64.3 \%)$ & $15.2-58.3 \%)$ \\
\hline
\end{tabular}

Abbreviation: $\mathrm{Cl}$, confidence interval.

Values of ${ }^{\mathrm{a}} 395.8$ and ${ }^{\mathrm{b}} 60.2 \mathrm{ng} / \mathrm{mL}$ corresponded to the 80 th percentile for peak and trough levels, respectively, and levels above these cut-offs were categorized as high for our analyses.

${ }^{c}$ Median peak and trough levels were 278.0 and $43.0 \mathrm{ng} / \mathrm{mL}$, respectively.

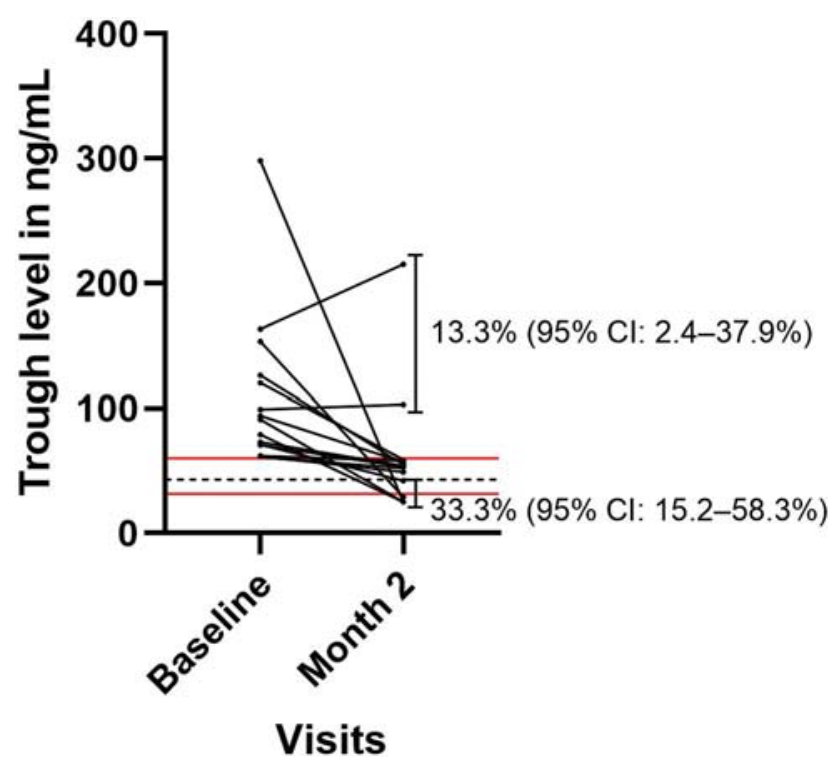

Fig. 3 Change in trough rivaroxaban levels across visits in patients with levels above the upper 20th centiles at baseline ( $\geq 60.2 \mathrm{ng} / \mathrm{mL}$ ). Solid, red horizontal lines represent the 20th and 80th percentiles of peak levels observed at baseline. Dashed, black horizontal lines represent the median trough level at baseline $(43.0 \mathrm{ng} / \mathrm{mL})$. Proportions of patients with levels remaining above or below the extreme 20th percentiles at month 2 are shown by the respective vertical capped lines. $\mathrm{Cl}$, confidence interval.

\section{Discussion}

We examined the possibility that an approach that measures rivaroxaban level once can be used to identify patients with persistently high rivaroxaban levels. We showed that at least two-thirds of patients with an initial peak or trough levels that fell above the respective 80th percentiles had subsequent levels that no longer fell in these extremes. The findings suggest that the measurement of a single peak or trough drug level cannot be used to reliably identify patients with persistently high rivaroxaban levels who might benefit from dose reduction.

Other studies have reported moderate intrapatient variability in rivaroxaban level based on an estimation of the $\mathrm{CV}$ of 
the study population but such measure is difficult to interpret clinically and does not directly apply to patients with extreme levels. ${ }^{8-12}$ Our findings are, however, consistent with those of Gulpen and colleagues who explored the intrapatient variability in postdose rivaroxaban level over 1 year using a similar approach to ours. ${ }^{13}$ Like us, they showed that a high proportion ( $\sim 60 \%$ ) of patients with an initial level that fell in the upper quintile subsequently had levels that were no longer high, but there were also some who had persistently high levels. Gulpen and colleagues concluded that their finding of a significant proportion of patients with persistently high or low drug levels offers the opportunity to adjust doses or switch to a different DOAC to enhance safety and efficacy of DOAC treatment. ${ }^{13}$ However, identification of patients potentially suitable for dose adjustment would require serial measurement of blood levels and before being accepted the efficacy and safety of such an approach would have to be demonstrated in a randomized trial.

Our findings suggest that a strategy based on single peak or trough rivaroxaban level measurement is not reliable to identify patients who should undergo a dose reduction. Furthermore, they raise the possibility for harm if the dose is reduced on the basis of one measurement because, on repeat testing, up to one-third of our patients had subsequent levels that were lower that the median drug level.

\section{Strengths and Limitations}

Our study has strengths and limitations. We carefully timed specimen collection to minimize preanalytical variability and examined variability in both trough and peak levels in patients with high drug levels. Potential limitations include the use of only one assay type to measure rivaroxaban levels, and the attrition of patients over time ( $20 \%)$, which may have reduced the precision of our estimates, although these findings highlight the practical challenges associated with patient adherence to a routine monitoring strategy. In addition, our study did not examine the determinants of variability in rivaroxaban level. Finally, our findings cannot exclude the possibility that multiple drug level measurements might identify patients who would benefit from a dose reduction. However, the net clinical benefit of such an approach requires further evaluation in randomized trials.

\section{Conclusion}

Our findings do not support the use of a single rivaroxaban level measurement to identify patients who would benefit from a dose reduction because such an approach is unable to reliably identify patients with high levels.

\section{Authors' Contributions}

N.C.C, J.H., J.S.G., and J.W.E. were involved in the conception and design of the project. K.S., N.C.C., and V.C.B. were involved in data analysis. All authors interpreted the data. K.S. prepared the initial version of the manuscript and interpretation. All authors contributed to the drafting and revising of the manuscript and approved the final version of the manuscript.

\section{Funding}

This study was funded by the Hamilton Health Sciences Corporation New Investigator Fund (grant no.: NIF13332), a competitive peer-reviewed grant to support research by early career investigators in Hamilton, Ontario, Canada. The funder was not otherwise involved in the study.

\section{Conflict of Interest}

V.C.B. has received grants from Pfizer, Canada, and honoraria from Bayer. J.W.E. has received honoraria and research support from Astra-Zeneca, Bayer, Boehringer Ingelheim, Bristol-Myers Squibb, Daiichi Sankyo, Janssen, Pfizer, Portola, and Sanofi. N.C.C. has received a speaker fee from Bayer outside the submitted work. The other authors report no conflicts of interests.

\section{References}

1 Lip GYH, Banerjee A, Boriani G, et al. Antithrombotic therapy for atrial fibrillation: CHEST guideline and expert panel report. Chest 2018;154(05):1121-1201

2 Kearon C, Akl EA, Ornelas J, et al. Antithrombotic therapy for VTE disease: CHEST guideline and expert panel report. Chest 2016; 149(02):315-352

3 Yeh CH, Fredenburgh JC, Weitz JI. Oral direct factor Xa inhibitors. Circ Res 2012;111(08):1069-1078

4 Food and Drug Administration. Rivaroxaban Clinical Review. Accessed 21 August 2020 at: http://www.accessdata.fda.gov/ drugsatfda_docs/nda/2011/2024390rig1s000MedR.pdf

5 Pollak PT, Mitchell LB. Monitoring direct oral anticoagulants: longing for the days when we were in control? Can J Cardiol 2019;35(06): 694-698

6 Chan N, Sager PT, Lawrence J, et al. Is there a role for pharmacokinetic/pharmacodynamic-guided dosing for novel oral anticoagulants? Am Heart J 2018;199:59-67

7 Eikelboom JW, Quinlan DJ, Hirsh J, Connolly SJ, Weitz JI. Laboratory monitoring of non-vitamin $\mathrm{K}$ antagonist oral anticoagulant use in patients with atrial fibrillation: a review. JAMA Cardiol 2017;2(05):566-574

8 Testa S, Tripodi A, Legnani C, et al; START-Laboratory Register. Plasma levels of direct oral anticoagulants in real life patients with atrial fibrillation: results observed in four anticoagulation clinics. Thromb Res 2016;137:178-183

9 Gulilat M, Tang A, Gryn SE, et al. Interpatient variation in rivaroxaban and apixaban plasma concentrations in routine care. Can J Cardiol 2017;33(08):1036-1043

10 Lin S-Y, Kuo C-H, Yeh S-J, et al. Real-world rivaroxaban and apixaban levels in Asian patients with atrial fibrillation. Clin Pharmacol Ther 2020;107(01):278-286

11 Miklič M, Mavri A, Vene N, et al. Intra- and inter- individual rivaroxaban concentrations and potential bleeding risk in patients with atrial fibrillation. Eur J Clin Pharmacol 2019;75 (08):1069-1075

12 Gouin-Thibault I, Delavenne X, Blanchard A, et al. Interindividual variability in dabigatran and rivaroxaban exposure: contribution of ABCB1 genetic polymorphisms and interaction with clarithromycin. J Thromb Haemost 2017;15(02):273-283

13 Gulpen AJW, Ten Cate H, Henskens YMC, et al. The daily practice of direct oral anticoagulant use in patients with atrial fibrillation; an observational cohort study. PLoS One 2019;14(06):e0217302 